\title{
Nuclear Effects in HERA-B
}

\author{
A. L. Ayala Filho, V. P. Gonçalves, \\ Instituto de Física e Matemática, Universidade Federal de Pelotas, \\ Campus Universitário s/no., Caixa Postal 354, 96010-900, Pelotas, RS, Brazil \\ and C. Brenner Mariotto \\ Departamento de Física, Fundação Universidade Federal do Rio Grande, \\ Rua Eng. Alfredo Huch, 475, 96201-900, Rio Grande, RS, Brazil
}

Received on 8 October, 2006

\begin{abstract}
In this contribution we estimate the influence of the shadowing effect and initial state parton energy loss in the quarkonium production at HERA-B. We analyze the $x_{F}$ behavior of the effective exponent $\alpha\left(x_{F}\right)$ and present a comparison with the preliminary HERA-B data for $J / \Psi$ production. Moreover, we present our predictions for $\Upsilon$ production at HERA-B. The magnitude of these effects in the $J / \Psi$ production at RHIC is also analyzed.
\end{abstract}

Keywords: Quarkonium production; Shadowing effects; Energy loss

The relativistic collider facilities RHIC and LHC will for the first time provide the opportunity to systematically study the physics of hot and ultradense matter in hadron-nucleus $(p A)$ and nucleus-nucleus $(A B)$ collisions at energies that are orders of magnitude larger than the energies of the current accelerators. The systematic study of $p A$ collisions at the same energies is essential to gain insight into the structure of the dense medium effects. Such effects, as the energy loss and shadowing, are absent or small in $p p$ collisions, but become increasingly prominent in $p A$ collisions, and are of major importance in $A A$ reactions. By comparing $p A$ and $A A$ reactions involving very heavy nuclei, one may be able to distinguish basic hadronic effects that dominate the dynamics in $p A$ collisions, from a quark-gluon formation predicted to occur in heavy ion $A A$ collisions. To gain insight into the underlying hadronic processes, one has to study collisions that are expected to not lead to a QGP formation. Once the physics of "QCD at high densities" is better understood, the mechanisms of quark-gluon plasma formation and related collective phenomena in heavy ion collisions could be disentangled from the basic hadronic effects.

In this contribution we study the influence of the nuclear medium effects in the quarkonium production, particularly of the $J / \psi$, which is one of the proposed signatures of the QCD phase transition [1]. In particular, we will consider the shadowing effects in the parton distributions and the initial state parton energy loss, which modify the $x_{F}\left(\equiv x_{1}-x_{2}\right)$ behavior of the cross section. We will focus our analyzes in the kinematical range of HERA-B, which recently has obtained data for the quarkonium production at negative values of $x_{F}$. As we will show, these data could be used to discriminate between the different models for the medium effects.

Lets start presenting a brief review about the nuclear medium effects. One of the nuclear medium effects is the nuclear shadowing, which is the modification of the target parton distributions so that $x q^{A}\left(x, Q^{2}\right)<A x q^{N}\left(x, Q^{2}\right)$, as expected from a superposition of $p p$ interactions. In recent years several experiments have been dedicated to high precision measurements of deep inelastic lepton scattering (DIS) off nuclei. Experiments at CERN and Fermilab focus espe- cially on the region of small values of the Bjorken variable $x=Q^{2} / 2 M v$, where $Q^{2}=-q^{2}$ is the squared four-momentum transfer, $v$ the energy transfer and $M$ the nucleon mass. The data $[2,3]$, taken over a wide kinematic range, have shown that the proton and neutron structure functions are modified by a nuclear environment. The modifications depend on the parton momentum fraction: for momentum fractions $x<0.1$ and $0.3<x<0.7$, a depletion is observed in the nuclear structure functions. The low $x$ (shadowing region) and the larger $x$ (EMC region) are bridged by an enhancement known as antishadowing for $0.1<x<0.3$. We refer to the entire phenomena as the nuclear shadowing effect.

The theoretical understanding of $F_{2}^{A}$ in the full kinematic region has progressed in recent years, with several models which describe the experimental data with quite success [4]. Here we will restrict ourselves to the descriptions which use the DGLAP evolution equations [5] to describe the behavior of the nuclear parton distributions. In particular, Eskola, Kolhinen and Salgado (EKS) have shown [6] that the experimental results [2] presenting nuclear shadowing effects can be described using the DGLAP evolution equations [5] with adjusted initial parton distributions. The basic idea of this framework is the same as in the global analyzes of parton distributions in the free proton: they determine the nuclear parton densities in a wide range of $x$ and $Q^{2} \geq Q_{0}^{2}=2.25 \mathrm{GeV}^{2}$ through their perturbative DGLAP evolution by using the available experimental data from $l A$ DIS and Drell-Yan (DY) measurements in $p A$ collisions as constraint. EKS have expressed the results in terms of the nuclear ratios $R_{f}^{A}\left(x, Q^{2}\right)$ for each parton flavor $f$ in a nucleus with $A$ nucleons $(A>2)$, at $10^{-6} \leq x \leq 1$ and $2.25 \mathrm{GeV}^{2} \leq Q^{2} \leq 10^{4} \mathrm{GeV}^{2}$. Other groups have considered different sets of data [7] and/or next-to-leading order corrections to the DGLAP equation [8] and proposed a distinct parameterization for the nuclear effects. In Fig. 1, taken from Ref. [8], we present a comparison between the different parameterizations for the nuclear ratios $R_{f}^{A}\left(x, Q^{2}\right)$ and $R_{g}^{A}\left(x, Q^{2}\right)$ at $A=C a$. We can see that these parameterizations predict very distinct behavior for the nuclear parton distributions. In particular, the magnitude of the anti-shadowing is still an open question. This scenario should be improved by the experi- 
mental analyzes of quarkonium production at HERA-B, since it probes the parton distributions in this $x$ range.

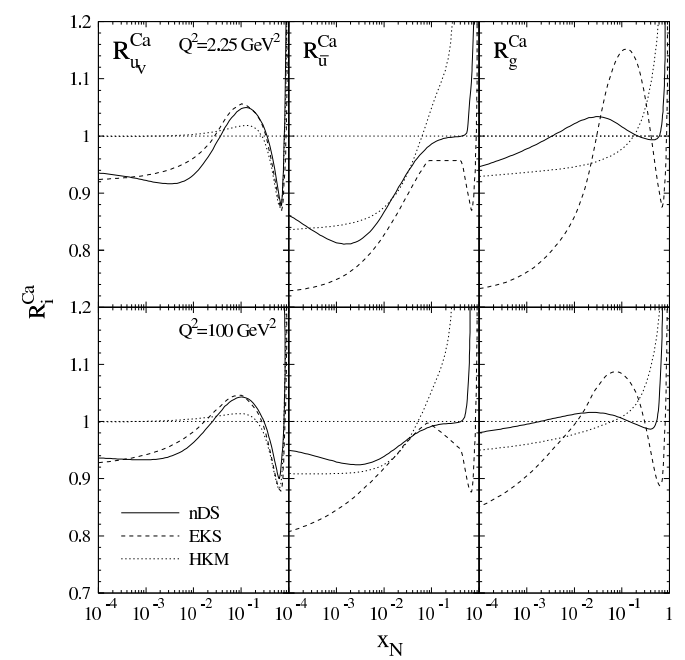

FIG. 1: Comparison between the distinct parameterization for the nuclear ratio $R_{f}^{C a}\left(x, Q^{2}\right)$ and $R_{g}^{C a}\left(x, Q^{2}\right)$. Figure taken from Ref. [8].

Another important effect in nuclear collisions is the initial state energy loss. It has been studied by Gavin and Milana (GM) [9] and subsequently developed by Brodsky and Hoyer [10] and Baier et. al. [11], considering a multiple scattering approach that essentially depletes the projectile parton momentum fraction, $x_{1}$, as the parton moves through the nucleus. Both the quarks and gluons can scatter elastically and loose energy before the hard scattering. The projectile parton momentum fraction involved in the hard scattering is then $x_{1}^{\prime}=x_{1}-\Delta x_{1}$ where $x_{1}$ is the original projectile parton momentum fraction when the parton first entered the target and $\Delta x_{1}$ represents the loss in $x_{1}$ due to multiple scatterings. Thus the shifted value, $x_{1}^{\prime}$, enters the partonic cross sections but the parton distributions must be evaluated at the initial $x_{1}$. In Ref. [11] the authors have demonstrated that the averaged radiative energy loss $-d E / d z$ is related to the characteristic squared transverse momentum of the parton $\left\langle p_{\perp W}^{2}\right\rangle$ by

$$
-\frac{d E}{d z}=\frac{3 \alpha_{s}}{4}\left\langle p_{\perp W}^{2}\right\rangle
$$

In this description, $\Delta x_{1}$ is then

$$
\Delta x_{1}=\frac{3 \alpha_{s}}{2} \frac{m_{p}}{x_{1} s} L_{A}\left\langle p_{\perp W}^{2}\right\rangle
$$

where the average transverse momentum $\left\langle p_{\perp W}^{2}\right\rangle$ is proportional to $A^{1 / 3}$ [11]. Since $\left\langle p_{\perp W}^{2}\right\rangle \propto A^{1 / 3}, \Delta x_{1} \propto A^{2 / 3}$ in Eq. (2) rather than $A^{1 / 3}$. It is important to emphasize that the Eq. (1) was derived using the eikonal picture to treat the propagation of a high energy parton transversing cold matter, assuming that the scattering centers are static and uncorrelated. The validity of these assumptions in the HERA-B kinematical range is still an open question.
Two estimates of $\left\langle p_{\perp W}^{2}\right\rangle$ were provided in Ref. [11]. The larger value, used as an upper limit, comes from a single nuclear rescattering of photoproduced dijets [12],

$$
\left\langle p_{\perp W}^{2}\right\rangle=\pi^{2} \alpha_{s} \lambda_{\mathrm{LQS}}^{2} A^{1 / 3} \frac{C_{A} \sigma_{g}^{\gamma A}+C_{F} \sigma_{q}^{\gamma A}}{\sigma^{\gamma A}} .
$$

They obtained $0.05<\lambda_{\mathrm{LQS}}^{2}<0.1 \mathrm{GeV}^{2}$ by assuming that dijet production is dominated either by quarks or gluons using the measured $p_{T}$ broadening as a function of $A$. With the lower bound on $\lambda_{\mathrm{LQS}}^{2}$,

$$
\left\langle p_{\perp W}^{2}\right\rangle \simeq 0.658 \alpha_{s} A^{1 / 3} \mathrm{GeV}^{2} .
$$

Since the initial states could not be explicitly identified, one assumes that $\left\langle p_{\perp W}^{2}\right\rangle$ is identical for quarks and gluons. Then when $\alpha_{s} \sim 0.3$ and $A=184$, one finds $-d E / d z \simeq 1.28$ $\mathrm{GeV} / \mathrm{fm}$ with Eq. (4). We refer to this as "LQS" in the remainder of the discussion. The second estimate depends on the nucleon gluon distribution and contains explicit color factors so that

$$
\begin{aligned}
& \left\langle p_{\perp W}^{2}\right\rangle_{q}=\frac{2 \pi^{2} \alpha_{s}}{3} \rho_{A} x G\left(x, Q^{2}\right) L_{A} \simeq 0.07 \alpha_{s} A^{1 / 3} \mathrm{GeV}^{2}(5) \\
& \left\langle p_{\perp W}^{2}\right\rangle_{g}=\frac{9}{4}\left\langle p_{\perp W}^{2}\right\rangle_{q} \simeq 0.15 \alpha_{s} A^{1 / 3} \mathrm{GeV}^{2}
\end{aligned}
$$

where $x G(x) \sim 1-2$ for the $x_{1}$ range of E866. This lower estimate is referred to subsequently as "BDMPS". Now when $\alpha_{s} \sim 0.3$ and $A=184,-d E_{q} / d z \simeq 0.12 \mathrm{GeV} / \mathrm{fm}$ and $-d E_{g} / d z \simeq 0.28 \mathrm{GeV} / \mathrm{fm}$.

In order to analyze the quarkonium production we will consider the color evaporation model (CEM) (see [13] for a more complete description). In this model, quarkonium production is treated identically to open heavy quark production except that the invariant mass of the heavy quark pair is restricted to be less than twice the mass of the lightest meson that can be formed with one heavy constituent quark. The LO charmonium cross section for charmonium state $i, \tilde{\sigma}_{i}$, is then obtained by integrating the free $c \bar{c}$ cross section over the pair mass from the $c \bar{c}$ production threshold, $2 m_{c}$, to the open charm threshold, $2 m_{D}=3.74 \mathrm{GeV}$. Then

$$
\frac{d \tilde{\sigma}_{i}}{d x_{F}}=2 F_{i} \int_{2 m_{c}}^{2 m_{D}} m d m \frac{d \sigma^{c \bar{c}}}{d x_{F} d m^{2}},
$$

where $F_{i}$ is the fraction of $\sigma^{c \bar{c}}$ that produces the final-state $c \bar{c}$ resonance. The CEM assumes that the quarkonium dynamics is identical to that of low invariant mass $c \bar{c}$ pairs. The hadronization of the charmonium states from the $c \bar{c}$ pairs is nonperturbative, involving the emission of one or more soft gluons. A different nonperturbative matrix element is needed for the direct production of each charmonium state. Each nonperturbative matrix element is represented by a single universal factor $F_{i}$ which depends on the charm quark mass, $m_{c}$, the scale of $\alpha_{s}, \mu$, and the parton densities. Once $F_{i}$ has been determined for each state, e.g. $\psi, \psi^{\prime}$ or $\chi_{c J}$, the model successfully predicts the energy and momentum dependencies. We note that $F_{\psi}$ includes both direct $\psi$ production and indirect production through radiative decays of the $\chi_{c J}$ states and 
hadronic $\psi^{\prime}$ decays. Also, $\frac{d \sigma^{c \bar{c}}}{d x_{F} d m^{2}}$ is the pQCD cross section convoluted with the parton distributions in the proton/nucleus, taken in LO for simplicity, since we are just interested in ratios of cross sections.

In order to investigate the medium dependence of the quarkonium production cross section, we will follow the usual procedure used to describe the experimental data on nuclear effects in the hadronic quarkonium production [14], where the atomic mass number $A$ dependence is parameterized by $\sigma_{p A}=\sigma_{p N} \times A^{\alpha}$. Here $\sigma_{p A}$ and $\sigma_{p N}$ are the particle production cross sections in proton-nucleus and proton-nucleon interactions, respectively. If the particle production is not modified by the presence of nuclear matter, then $\alpha=1$. A number of experiments have measured a less than linear $A$ dependence for various processes of production, which indicates that the medium effects cannot be disregarded. To estimate the modification of quarkonium production cross section due to the medium effects, we calculate the effective exponent $\alpha\left(x_{F}\right)$, which is given by

$$
\alpha\left(x_{F}\right)=\left\{\ln \left(\frac{d \sigma_{p A}}{d x_{F}} / \frac{d \sigma_{p N}}{d x_{F}}\right) / \ln A\right\} .
$$

Moreover, to obtain the available observable measured in the experiments, we also replace the nucleon by a light nucleus target (carbon), calculating the ratio

$$
\alpha_{A_{2} / A_{1}}\left(x_{F}\right)=\left\{\ln \left(\frac{d \sigma_{p A_{2}}}{d x_{F}} / \frac{d \sigma_{p A_{1}}}{d x_{F}}\right) / \ln \left(A_{2} / A_{1}\right)\right\},
$$

where $A_{1}=C$ and $A_{2}=P d, W$.

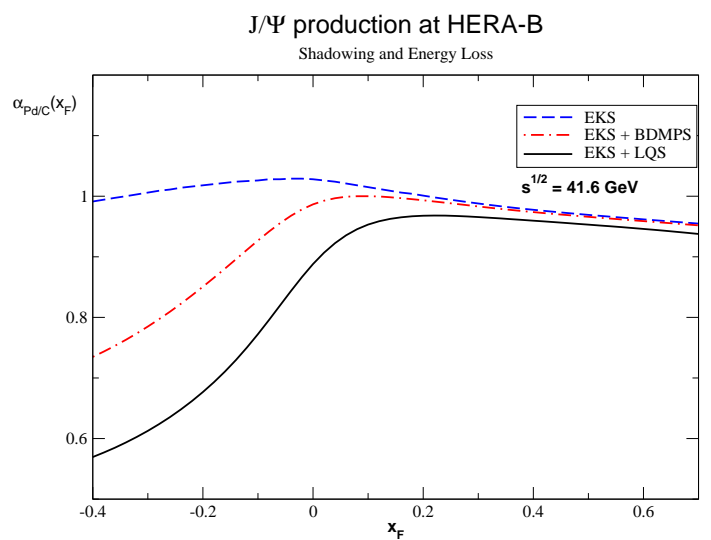

FIG. 2: Effective exponent as a function of $x_{F}$ for charmonium production at HERA-B considering palladium and carbon targets.

Our results for the effective exponent $\alpha_{A_{2} / A_{1}}\left(x_{F}\right)$ in $J / \Psi$ production at HERA-B energy of $\sqrt{s}=41.6 \mathrm{GeV}$, with palladium and carbon targets are shown in Fig. 2. In the dashed curve only the nuclear shadowing effects are taken into account through the EKS parameterization. Energy loss effects

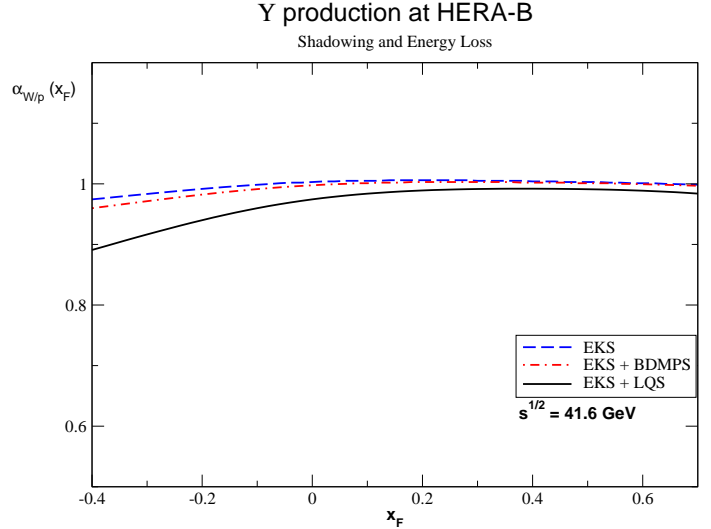

FIG. 3: Effective exponent as a function of $x_{F}$ for bottomonium production at HERA-B considering tungsten and proton targets.

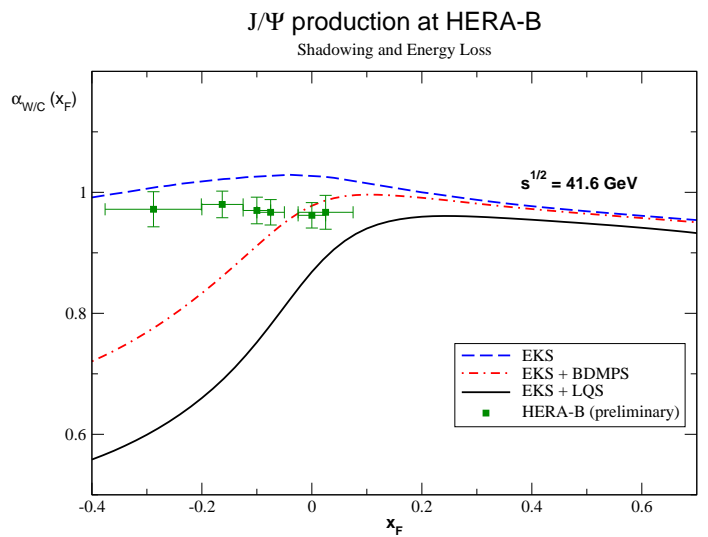

FIG. 4: Effective exponent as a function of $x_{F}$ for charmonium production at HERA-B considering tungsten and carbon targets.

are also included in the dot-dashed (BDMPS estimate) and full (LQS estimate) curves. Whereas the shadowing alone produces a small enhancement for negative $x_{F}$ (antishadowing indeed) and a small suppression for higher positive values of $x_{F}$, the inclusion of energy loss leads to a large suppression for the heavy nuclear target, for negative values of $x_{F}$. This suppression can be understood in the models, since the projectil parton looses its momentum when traversing the nuclear target.

Results for the effective exponent in bottomonium production at HERA-B energies are shown in fig. 3. As we can see, the suppression effect is quite small compared to the $J / \Psi$ case. This behavior is associated to the $x$ range probed in this process.

In Fig. 4 we present our results for $J / \Psi$ production at same energies, in a tungsten and carbon targets, compared with preliminary HERA-B data for $J / \Psi$ production [15]. Neither the enhancement due to the shadowing effects alone, nor the strong suppression for $x_{F}<0$ due to energy loss are seen in the data, which indicate a small and $x_{F}$-independent suppression. These results may indicate that the magnitude of the anti-shadowing is overestimated in the EKS parameterization or that the energy loss is smaller that predicted by the LQS 
and BDMPS models. In the latter case, it can be associated with the breakdown of the basic assumptions of the BDMPS formalism in the HERA-B kinematical range.

Another possibility to constrain the magnitude of the medium effects is the study of the quarkonium production in proton(deuteron)-nucleus collisions at RHIC. In this case, due to the larger center of mass energy, the nuclear effects should be amplified. In Fig. 5 we present our estimates for the effective exponent for $J / \Psi$ production at $\sqrt{s}=200 \mathrm{GeV}$ RHIC energies, with proton and gold targets. As we can see, the effective exponent is almost $x_{F}$ independent in the positive $x_{F}$ range. On the other hand, the energy loss leads to a very strong suppression for negative $x_{F}$, larger than that predicted at HERA-B. It implies that the experimental analysis of the quarkonium production is ideal to constrain the initial state energy loss effects.

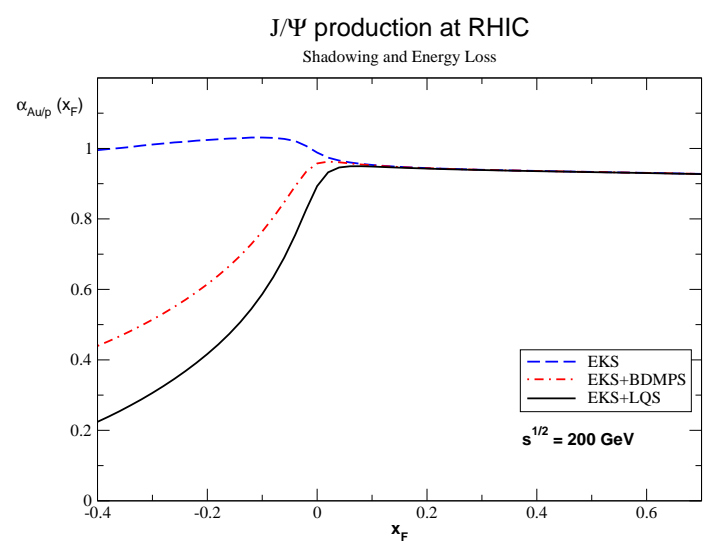

FIG. 5: Effective exponent as a function of $x_{F}$ for charmonium production at RHIC considering gold and proton targets.

As a summary, in this contribution we have studied the quarkonium production at HERA-B and RHIC. In particular, we have considered the shadowing and energy loss effects and analyzed the $x_{F}$ behavior of the effective exponent. Our main emphasis was in the negative $x_{F}$ range which have been probed at HERA-B. We have verified that the inclusion of the energy loss strongly modify the behavior of $\alpha\left(x_{F}\right)$ in this kinematical range. The comparison of our predictions with the preliminary data indicates that the combination of shadowing and energy loss effects, as described by the EKS parameterization and LQS or BDMPS models, is not able to describe the data. This may be related to the applicability of the BDMPS approach in cold matter and/or the overestimation of the antishadowing effect in the EKS parameterization. One effect which we have disregarded in our analyzes was the nuclear absorption which is associated with the fact that the $c \bar{c}$ pair may interact with nucleons and be dissociated or absorbed before it can escape the target. However, the inclusion of this effect in combination with shadowing and energy loss effects will implicate a larger suppression at negative $x_{F}$, which is disfavoured by the data. On the other hand, if only this effect is considered the data can be reasonably described [16], which turns the estimate of the nuclear effects in quarkonium production an open question. Another uncertainty present is the validity of the collinear factorization in nuclear collisions. Some authors advocate that the coherence phenomena cannot be disregarded [17].

Since the suppression predicted in RHIC is larger, it would be desirable to have measurements in that region to constrain the different models and disentangle the different effects. To conclude, nuclear collisions remain a fascinating place to study different effects including the interplay of perturbative and non-perturbative QCD, and nuclear effects.

Ackowledgements. This work was partially financed by CNPq and FAPERGS, Brazil.
[1] T. Matsui, H. Satz, Phys. Lett. B 178, 416 (1986).

[2] M. Arneodo et al. Nucl. Phys. B 483, 3 (1997); Nucl. Phys. B 441, 12 (1995).

[3] M. R. Adams et al.. Z. Phys. C 67, 403 (1995).

[4] See, e.g., M. Arneodo. Phys. Rep. 240, 301 (1994); G. Piller, W. Weise. Phys. Rep. 330, 1 (2000).

[5] Yu. L. Dokshitzer. Sov. Phys. JETP 46, 641 (1977); G. Altarelli and G. Parisi. Nucl. Phys. B 126, 298 (1977); V. N. Gribov and L. N. Lipatov. Sov. J. Nucl. Phys 15, 438 (1972).

[6] K. J. Eskola, V. J. Kolhinen, and C. A. Salgado. Eur. Phys. J. C 9, 61 (1999); K. J. Eskola, V. J. Kolhinen, and P. V. Ruuskanen. Nucl. Phys. B 535, 351 (1998).

[7] M. Hirai, S. Kumano, and M. Miyama, Phys. Rev. D 64, 034003 (2001); M. Hirai, S. Kumano, and T. H. Nagai, Phys. Rev. C 70, 044905 (2004).

[8] D. de Florian and R. Sassot, Phys. Rev. D 69, 074028 (2004).

[9] S. Gavin and J. Milana, Phys. Rev. Lett. 68, 1834 (1992).

[10] S.J. Brodsky and P. Hoyer, Phys. Lett. B 298, 165 (1993).
[11] R. Baier, Yu.L. Dokshitser, A.H. Mueller, S. Peigne, and D. Schiff, Nucl. Phys. B 484, 265 (1997); R. Baier, Yu.L. Dokshitser, A.H. Mueller, and D. Schiff, Nucl. Phys. B 531, 403 (1998).

[12] M. Luo, J. Qiu, and G. Sterman, Phys. Rev. D 49, 4493 (1994).

[13] C. B. Mariotto, M. B. Gay Ducati, and G. Ingelman, Eur. Phys. J. C 23, 527 (2002); J. F. Amundson, et al., Phys. Lett. B 390, 323 (1997); M. B. Gay Ducati, V. P. Goncalves, and C. B. Mariotto, Phys. Rev. D 65, 037503 (2002)

[14] See, e. g., M. J. Leich et al., Nucl. Phys. A 544, 197c (1992).

[15] U. Husemann, Measurement of nuclear effects in the production of $J / \Psi$ mesons with the HERA-B detector, Dissertation, DESY report: DESY-THESIS-2005-005.

[16] R. Vogt, Nucl. Phys. A 700, 539 (2002).

[17] B. Kopeliovich, A. Tarasov, and J. Hufner, Nucl. Phys. A 696, 669 (2001) 